\title{
Approximations, Expansions and Univalued Representations of Multifunctions *
}

\author{
Darinka Dentcheva \\ Humboldt-Universität Berlin \\ 10099 Berlin, Germany \\ dary@mathematik.hu-berlin.de
}

\begin{abstract}
We consider multifunctions acting between two linear normed spaces and having closed convex images. Approximations are considered which serve as an expansion of it. Generalized delta theorems for random sets in infinite dimensions are shown using those approximations. Furthermore, univalued, resp. Castaing representations of the multifunction are constructed with (higher order) differentiability properties at certain points whose directional derivative form a univalued, resp. Castaing representation of the corresponding "derivative" of the multifunction. The construction yields higher order information about the asymptotic distributions of measurable selections forming the Castaing representation of the multifunction.
\end{abstract}

Keywords: Higher-order differentiable multifunctions, selections, Wijsman topology, Castaing representation, delta theorems

\section{Introduction}

Throughout the paper, we shall consider a multifunction $F: X \rightrightarrows Y$ acting between two linear normed spaces $X$ and $Y$.

There are nowadays several concepts of generalized differentiability of multifunctions introduced with various purposes. Differentiability is defined by introducing a class of approximating mappings. There are two aspects of the definition: the kind of approximations and the choice of topologies. Let us mention some of the papers on the subject proposing such notions. In [6] the approximating multifunction is in some sense affine and continuous. In [16]. the approximations are upper semi-continuous and homogeneous, and have bounded closed convex images. In [23], "affine" approximating multifunctions for the case $Y=\mathbb{R}^{n}$ are suggested. Lemarechal and Zowe ([28])

*Supported by HSP III Program of Humboldt University Berlin 
define eclipsing multifunctions as approximations. Those concept are investigated and compared in [22] by Gautier. Several other concepts of differentiability represent some cone-approximation of the multifunction. Furthermore, corresponding higher order tangent sets have been developed. Some geometrical concepts are presented in [4], which correspond to looking at certain tangent cone to the graph of the multifunction. A stronger differentiability notion related to interesting phenomena in optimization, called proto-differentiability, has been introduced by Rockafellar in [32]. Another attractive concept of differentiability called semi-differentiability. This concept goes back to Penot [31] and corresponds to a concept of tangential approximation due to Shapiro, investigated in various papers [37, 38, 39].

Our aim is to introduce approximations that serve as local directional expansions of the multifunction. The concept presents an extension of the notion semi-differentiability The notion is modified by using a metric convergence on the hyperspace of nonempty closed subsets of $Y$, which is better suited to the infinite-dimensional case. This differentiability property is well investigates in finite dimensional case. We have to emphasize that, if the image space $X$ is finite-dimensional, it is still a slight modification of the concept of semi-differentiability by Penot. The first order approximation is a closed continuous process with respect to the direction.

The introduced concept of differentiability becomes useful while dealing with asymptotic behavior of random sets. One of the most widely used approaches to gain information on the the asymptotic behavior of random elements is the delta method. Generally speaking, delta theorems establish central limit formulas for random elements in complete metric spaces. There is a set-valued version of a delta theorem (see [25]) dealing with random sets in $\mathbb{R}^{n}$. We shall prove such a theorem for random sets in Polish spaces. Moreover, we prove also a generalization, which deals with higher order central limit formulas.

Further goal of this paper is to construct a representation of the multifunction by countably many selections of it, such that for a dense set of points of the graph of the multifunction there is a selection that meets the point and has a directional expansion at the point. To this end we construct selections with higher order directional differentiability properties, using the introduced tangential approximations of the mapping. A mapping $f: X \rightarrow Y$ is a selection of $F$ if $f(x) \in F(x)$ for all $x \in X$. By the axiom of choice, such a mapping exists whenever $F$ has non-empty images. One is usually interested in finding selections with additional properties. The question of existence of selections with differentiability properties is of interest in its own. Investigations on this topic are presented in $[17,20,23]$. Gautier and Morchadi consider the so-called Steiner selection and its remarkable properties in [23]. Conditions for differentiability properties of Steiner selections are identified also in [17, 20]. In [20], Dommisch exploits the existence of a smooth partition of unity in $\mathbb{R}^{n}$ to prove the existence of a smooth selection under some additional conditions. We shall see that one of her constructions 
carries over to the case of infinite dimensions.

The Steiner center of convex sets is well-defined in finite dimensional spaces only. It is shown in [41] that some natural attempts to generalize the definition for sets in infinite dimensional spaces have unsatisfactory results. This is the reason why we try to obtain selections by using another approach.

The constructed representation is of interest while dealing with measurable multifunction. It is well-known, that the measurability of a multifunction is characterized by the existing of a countable family of measurable selections that pointwise fill up the images of the multifunction. Such a family is called a Castaing representation. Our construction yields higher order information about the asymptotic distributions of measurable selections forming a Castaing representation of the multifunction. Moreover, the first-order directional derivative of the selections form a Castaing representation of the first-order approximation of the multi-function at the points of interest.

This paper presents a generalization of some of the results in [17] for mappings valued in infinite dimensions. One generalization is the (higher order) tangential approximation of the graph of the multifunction, and the construction of a continuous selection that has a directional expansion at given points. These results lead to a construction of a Castaing representation with higher order differentiability properties. An important property of the construction of the Castaing representation is that the directional derivative (of any order) of the selections form a Castaing representation of the corresponding semi-derivatives at certain points. Correspondingly, higher order delta theorems for the members of these Castaing representation are shown.

The paper is organized as follows. In section 2, we formulate the necessary notions, recall some facts and show some basic properties of the approximations. We also make a brief review of some other differentiability concepts.

In section 3, a construction of selections is presented. It represents a selection which is Hadamard-directionally differentiable up to given order $k$ at certain points. In section 4, a univalued representation of the multifunction is presented. In section 5, delta theorems and convergence in distribution for random sets and their selections are discussed.

\section{Differentiability of multifunctions}

Throughout the whole paper we will consider set-valued mappings $F: X \rightrightarrows Y$ defined on a linear normed space $X$ with nonempty closed convex values in a linear normed space $Y$. These investigations can be extended also for a multifunctions with nonempty closed domain adapting all notions to the restriction of the multifunction to its domain. We denote the graph of $F$ by graph $F$.

Let $\mathcal{F}(Y)$ denote the hyperspace of nonempty closed sets in $Y$, and let $\mathcal{F}_{c}(Y)$ stand 
for the corresponding hyperspace of nonempty closed convex sets. We endow these spaces with the Wijsman topology determined by the metric $d(x, y)=\|x-y\|$ and denoted by $\tau$. Abusing notation we shall denote the distance of a point $y \in Y$ to a nonempty closed set $A \subset Y$ by $d(y, A)$, i.e., $d(y, A)=i n f_{z \in A} d(y, z)$. By definition $\tau$ is the weak topology determined by the family $\{d(y, \cdot): y \in Y\}$. We recall some basic facts concerning the topological space $(\mathcal{F}(Y), \tau)$ and refer to $[7,8]$ for further details. A subbase of $\tau$ consists of all sets of the form:

$$
\{A \in \mathcal{F}(Y): d(y, A)<\varepsilon\} \text { and }\{A \in \mathcal{F}(Y): d(y, A)>\varepsilon\} \quad(y \in Y, \varepsilon>0) \text {. }
$$

A sequence of closed sets $\left\{A_{n}\right\}, A_{n} \subseteq Y$ converges to some $A \subseteq Y$ in $(\mathcal{F}(Y), \tau)$ (written $\left.A_{n} \stackrel{W}{\rightarrow} A\right)$ if and only if the sequence of the distance functions converges pointwise, i.e.,

$$
A_{n} \stackrel{W}{\rightarrow} A \quad \text { if and only if } \quad d\left(y, A_{n}\right) \rightarrow d(y, A) \text { for all } y \in Y,
$$

The space $(\mathcal{F}(Y), \tau)$ is metrizable if and only if $Y$ is separable. For $\left\{y_{i}\right\}$ being a countable dense subset of the separable metric space $(Y, d)$ the following metric is compatible with the Wijsman topology:

$$
\rho_{d}(A, B)=\sum_{i=1}^{\infty} 2^{-i} \min \left\{1,\left|d\left(y_{i}, A\right)-d\left(y_{i}, B\right)\right|\right\} .
$$

Moreover, if $(Y, d)$ is a complete separable metric space, then $(\mathcal{F}(Y), \tau)$ is a Polish space, i.e., it is separable and there is a complete metric compatible with the topology (cf. [9]).

Our approximating multifunctions will be based on the following notions of differentiability:

Definition 2.1 A mapping $F: X \rightrightarrows Y$ is called directionally differentiable at a point $(x, y) \in \operatorname{graph} F$ in direction $h \in X$, if for any sequence $t_{n} \downarrow 0$ the limit

$$
F^{\prime}(x, y ; h)=\lim _{t_{n} \downarrow 0} \frac{F\left(x+t_{n} h\right)-y}{t_{n}}
$$

exists in $(\mathcal{F}(Y), \tau)$.

The original definition of semi-differentiability (cf.[31]) is modified in the following way:

Definition 2.2 A mapping $F: X \rightrightarrows Y$ is called semi-differentiable at a point $(x, y) \in$ graph $F$, if for all sequences $t_{n} \downarrow 0$ and $h_{n} \rightarrow h_{0}$ the limit

$$
D F(x, y)\left(h_{0}\right)=\lim _{t_{n} \downarrow 0, h_{n} \rightarrow h_{0}} \frac{F\left(x+t_{n} h_{n}\right)-y}{t_{n}}
$$

exists in $(\mathcal{F}(Y), \tau)$ for all $h_{0} \in X$. 
The convergence $h_{n} \rightarrow h_{0}$ is understood as the norm-convergence in $X$. We recall that, originally, these two notions are associated with the Kuratowski-Painlevé convergence of the corresponding differential quotients in the literature. For $Y$ being infinite dimensional, there is no topologization of this convergence in general (cf.[7]). This means that, in general, there is no topology on $\mathcal{F}(Y)$ such that the convergence of elements of $\mathcal{F}(Y)$ in the sense of Kuratowski-Painlevé will force convergence with respect to the topology. We require some stronger convergence in the definitions above. The Wijsman topology is a natural counterpart of the Kuratowski-Painlevé convergence for infinite dimensional spaces. Moreover, it plays a crucial role for measurability properties and convergence in distribution of random sets as we shall see later. Both convergences coincide if and only if $Y$ is finite dimensional.

The various notions of first order differentiability are extended to higher order properties e.g. in $[3,4,36]$. The approach used to introduce a higher order derivative is to define sets that are higher order "tangent" to the graph of the multifunction. This way of introducing second-order derivatives typically leads to a set-valued counterpart of second order directional derivative in the sense of Ben-Tal and Zowe. Let us mention the notion of first and second order pseudo-differentiability considered in [3]. Since the Kuratowski-Painlevé convergence of closed sets in $\mathbb{R}^{n}$ is characterized by the convergence of the distance function, it is shown in [3] that $A$ is pseudo-differentiable at $x$ if the distance function $d(\cdot, A)$ is directionally differentiable, and $A$ is twice pseudodifferentiable if $d(\cdot, A)$ admits a second order directional derivative in the sense of Ben-Tal and Zowe, i.e., setting $d_{A}(\cdot)=d(\cdot, A)$

$$
d_{A}^{\prime \prime}(x ; h, v)=\lim _{t \downarrow 0} \frac{d_{A}\left(x+t h+t^{2} v / 2\right)-d_{A}(x)-t d_{A}^{\prime}(x ; h)}{t^{2} / 2} .
$$

The second order semi-differentiability is considered there in a similar way, i.e., the multifunction $F: \mathbb{R}^{m} \rightrightarrows \mathbb{R}^{n}$ is called twice semi-differentiable at $(x, y)$ in direction $(h, z)$ if the limit

$$
\lim _{t \downarrow 0, v \rightarrow v_{0}} \frac{F\left(x+t h+t^{2} v / 2\right)-y-t z}{t^{2} / 2}
$$

exists for all $v_{0} \in \mathbb{R}^{m}$.

We shall use another concept, which will turn useful for the application we have in mind.

Let $f: X \rightarrow Y$ be single-valued mapping. Many papers (see, e.g., [?, ?]) consider natural to introduce higher order directional differentiability of $f$ at some point $x$, if it has a higher order expansion at that point into the direction under consideration, corresponding to the Taylor's expansion. We adopt the same approach and use the following:

Definition 2.3 Let $f: X \rightarrow Y$ be single-valued mapping. It is called directionally differentiable of order $k$ at the point $x$ into a direction $\bar{h}$, if the directional derivative

$$
f^{(k)}(x ; \hat{h}):=\lim _{t \downarrow 0, h \rightarrow \hat{h}} \frac{f(x+t h)-f(x)-t f^{(1)}(x ; h)-\ldots-t^{k-1} f^{(k-1)}(x ; h)}{t^{k}}
$$


up to order $1, \ldots, k$ at that point exists.

The same way we introduce a set-valued version of the notion. Since the derivatives are multivalued, it is natural to replace $f^{(k)}(x ; h)$ in the definition by some element $z^{k}$ of the corresponding set, which represents the semi-derivative. We shall use the notations:

$$
\Delta^{k+1} F(x, y)\left(v_{n}\right):=\frac{F\left(x+t_{n} h_{n}\right)-y-t_{n} z_{n}^{1}-t_{n}^{2} z_{n}^{2}-\ldots-t_{n}^{k} z_{n}^{k}}{t_{n}^{k+1}}
$$

for the $k$-th differential quotient, where $v_{n}$ stand for a proper direction, defined by the following setting:

$$
v_{n}=\left(h_{n}, z_{n}^{1}, \ldots, z_{n}^{k}\right), \text { where }\left(h_{n}, z_{n}^{i}\right) \in \operatorname{graph} D^{i} F(x, y)
$$

Definition 2.4 The mapping $F: X \rightrightarrows Y$ is called semi-differentiable of order $k+1$, $k=1,2, .$. at a point $(x, y) \in$ graph $F$ if for all sequences $t_{n} \downarrow 0$ and $v_{n} \rightarrow v$, where $v_{n}$ and $v$ are directions as in (1). the limit

$$
D^{k+1} F(x, y)\left(v_{n}\right)=\lim _{t_{n} \downarrow 0, v_{n} \rightarrow v} \Delta^{k+1} F(x, y)\left(v_{n}\right)
$$

exists in $(\mathcal{F}(Y), \tau)$ for all directions $v$ of the form (1).

The convergences $h_{n} \rightarrow h$ and $z_{n}^{i} \rightarrow z^{i}$ are taken with respect to the corresponding norms. This definition is sensible only if for all $z^{i} \in D^{i} F(x, y)(h)$, there are elements $z_{n}^{i} \in D^{i} F(x, y)\left(h_{n}\right)$ that converge in norm to $z^{i}$ while $h_{n} \rightarrow h$. As a matter of fact, there exist such elements by virtue of Proposition 2.6 bellow.

Let us observe that a multifunction is semi-differentiable of order $k$ at a certain point if the corresponding limit exists and is, in addition, nonempty. This requirement is implicitly included in the definitions above since only nonempty subsets are included in $\mathcal{F}(Y)$ and we consider the convergence there. Originally, the existence of the limit with respect to the Kuratowski-Painlevé convergence does not guarantee nonempty derivatives. It is evident that the semi-derivatives of all orders are closed sets. Recall that a multifunction is called closed if its graph is closed. It is called a process if its graph is a cone, i.e., it is positive homogeneous. We shall show by the next two propositions that the first order semi-derivative is a closed continuous process as a multifunction of the direction. Furthermore, the higher order semi-derivative is also closed,continuous, and satisfies a generalized condition for positive homogeneity.

Proposition 2.5 The multifunctions $D^{i} F(x, y)(\cdot), i=1,2, \ldots$ have the following properties:

(a) If $F(x)$ are convex sets for all $x$ in a neighborhood of $\bar{x}$, then the semi-derivatives at $(\bar{x}, y), y \in F(\bar{x})$ have convex images as well; 
(b) $z \in D^{i} F(x, y)\left(h, z^{1}, \ldots, z^{i-1}\right)$ if and only if $\lambda^{i} z \in D^{i} F(x, y)\left(\lambda h, \lambda z^{1}, \ldots, \lambda^{i-1} z^{i-1}\right)$, i.e., $D F(x, y)$ is a process.

Proof: Let $u, w \in D^{i} F(x, y)\left(h, z^{1}, \ldots, z^{i-1}\right)$ and $\lambda \in(0,1)$. According to the definition, given sequences $t_{n} \downarrow 0$ and $v_{n}:=\left(h_{n}, z_{n}^{1}, \ldots, z_{n}^{i-1}\right) \rightarrow v=\left(h, z^{1}, \ldots, z_{n}^{i-1}\right)$, where $\left(h_{n}, z_{n}^{k}\right),\left(h, z^{k}\right) \in \operatorname{graph} D^{k} F(x, y)$ for all $k=1, \ldots, i$, we obtain that both:

$$
d\left(u, \Delta^{i} F(x, y)\left(h_{n}, z_{n}^{1}, \ldots, z_{n}^{i-1}\right)\right) \text { and } d\left(w, \Delta^{i} F(x, y)\left(h_{n}, z_{n}^{1}, \ldots, z_{n}^{i-1}\right)\right)
$$

converge to 0 . Consequently, there are sequences $u_{n}$ and $w_{n}$ such that $u_{n}, w_{n} \in$ $\Delta^{i} F(x, y)\left(h_{n}, z_{n}^{1}, \ldots, z_{n}^{i-1}\right)$ and $u_{n} \rightarrow u, w_{n} \rightarrow w$. Since $F\left(x+t_{n} h_{n}\right)$ is convex, the points $\lambda\left(y+t_{n} z_{n}^{1}+\ldots+t_{n}^{i-1} z_{n}^{i-1}\right)+t_{n}^{i} u_{n}+(1-\lambda)\left(y+t_{n} z_{n}^{1}+\ldots+t_{n}^{i-1} z_{n}^{i-1}+t_{n}^{i} w_{n}\right) \in$ $F\left(x+t_{n} h_{n}\right)$. Consequently, $\lambda u_{n}+(1-\lambda) w_{n} \in \Delta^{i} F(x, y)\left(h_{n}, z_{n}^{1}, \ldots, z_{n}^{i-1}\right)$. Hence, for all $y \in Y$

$$
\begin{aligned}
& d\left(\lambda u+(1-\lambda) w, D^{i} F(x, y)\left(h_{n}, z_{n}^{1}, \ldots, z_{n}^{i-1}\right)\right)= \\
& \lim _{n \rightarrow \infty} d\left(\lambda u+(1-\lambda) w, \Delta^{i} F(x, y)\left(h_{n}, z_{n}^{1}, \ldots, z_{n}^{i-1}\right)\right) \leq \\
& \lim _{n \rightarrow \infty} d\left(\lambda u+(1-\lambda) w, \lambda u_{n}+(1-\lambda) w_{n}\right)+ \\
& \lim _{n \rightarrow \infty} d\left(\lambda u_{n}+(1-\lambda) w_{n}, \Delta^{i} F(x, y)\left(h_{n}, z_{n}^{1}, \ldots, z_{n}^{i-1}\right)\right)=0
\end{aligned}
$$

This proves (a).

The second assertion will be proved by induction. Let $z \in D F(x, y)(h)$. By definition $\lambda z \in D F(x, y)(\lambda h)$, if $d\left(\lambda z, t_{n}^{-1}\left(F\left(x+t_{n} \lambda h_{n}\right)-y\right)\right)$ converges to 0 while $n \rightarrow \infty$. Denoting $\hat{t}_{n}=\lambda t_{n}$, we have:

$$
d\left(\lambda z, t_{n}^{-1}\left(F\left(x+t_{n} \lambda h_{n}\right)-y\right)\right)=\lambda d\left(z, \hat{t}_{n}^{-1}\left(F\left(x+\hat{t}_{n} h_{n}\right)-y\right)\right)
$$

Since $\hat{t}_{n} \downarrow 0$ it holds $d\left(z, \hat{t}_{n}^{-1}\left(F\left(x+\hat{t}_{n} h_{n}\right)-y\right)\right) \rightarrow 0$ and this proves the assertion for the case $i=1$. Let us assume that it is true for $i \leq k$. Given $z \in D^{k+1} F(x, y)\left(h, z^{1}, \ldots, z^{k}\right)$ we have to prove that $\lambda^{k+1} z \in D^{k+1} F(x, y)\left(\lambda h, \lambda z^{1}, \ldots, \lambda^{k} z^{k}\right)$ By the induction assumption $\lambda^{i} z_{i} \in D^{i} F(x, y)\left(\lambda h, \lambda z^{1}, \ldots, \lambda^{i-1} z^{i-1}\right)$ for $i \leq k$. Denoting $\hat{t}_{n}=\lambda t_{n}$, we have:

$$
\begin{aligned}
& d\left(\lambda^{k+1} z, t_{n}^{-k-1}\left(F\left(x+t_{n} \lambda h_{n}\right)-y-t_{n} \lambda z_{n}^{1}-\ldots-t_{n}^{k} \lambda^{k} z_{n}^{k}\right)\right)= \\
& \lambda^{k+1} d\left(z, \hat{t}_{n}^{-k-1}\left(F\left(x+\hat{t}_{n} h_{n}\right)-y-\hat{t}_{n} z_{n}^{1}-\ldots-\hat{t}_{n}^{k} z_{n}^{k}\right)\right)=
\end{aligned}
$$

The righhand-side converges to 0 by definition. This completes the proof.

The statement $[\mathrm{b}]$ is a counterpart of the properties of the higher order contingent sets, considered in [4], Section 4.7.

We will show that the semi-derivatives are $\tau$-continuous multifunctions of the directions and, thus, represent a continuous approximation of the multifunction. Correspondingly, the $k$-th order derivatives are $\tau$-continuous with respect to the direction $\left(h, z^{1}, \ldots, z^{k-1}\right)$. 
We call a multifunction $F: X \rightrightarrows Y \tau$-continuous at a point $\bar{x} \in X$ if for all sequences $x_{n} \rightarrow \bar{x}$

$$
\tau-\lim _{x_{n} \rightarrow \bar{x}} F\left(x_{n}\right)=F(\bar{x}) .
$$

Proposition 2.6 Let $F: X \rightrightarrows Y$ be semi-differentiable of order $k$, $(k \geq 1)$ at $(\bar{x}, \bar{y})$. Then, the semi-derivative is a closed and $\tau$-continuous multifunction of the direction, i.e.,

$$
D^{k} F(\bar{x}, \bar{y})(v)=\tau-\lim _{n \rightarrow \infty} D^{k} F(\bar{x}, \bar{y})\left(v_{n}\right)
$$

where $v_{n}, v$ are directions according to the notation (1) and $v_{n} \rightarrow v$.

Proof: Let a sequence $h_{n} \rightarrow h$ be given. We consider $d\left(y, D F(\bar{x}, \bar{y})\left(h_{n}\right)\right)$ for arbitrary but fixed $y \in Y$. Given an $\varepsilon>0$, then there exists a positive number $t_{n}$ (that can be taken smaller than $t_{n-1}$ ) for any $n$ such that

$$
\left|d\left(y, D F(\bar{x}, \bar{y})\left(h_{n}\right)\right)-d\left(y, t_{n}^{-1}\left(F\left(\bar{x}+t_{n} h_{n}\right)-\bar{y}\right)\right)\right| \leq \varepsilon
$$

by the definition of a semi-derivative and $\tau$-convergence. For the same reason the following inequality holds for $n$ large enough:

$$
\left|d(y, D F(\bar{x}, \bar{y})(h))-d\left(y, t_{n}^{-1}\left(F\left(\bar{x}+t_{n} h_{n}\right)-\bar{y}\right)\right)\right| \leq \varepsilon .
$$

Consequently, $\left|d(y, D F(\bar{x}, \bar{y})(h))-d\left(y, D F(\bar{x}, \bar{y})\left(h_{n}\right)\right)\right| \leq 2 \varepsilon$ for $n$ large enough. Letting $\varepsilon \rightarrow 0$ we obtain the convergence of the distance functions and the continuity of the first-order semi-derivative.

Let now an element $\bar{z}^{1} \in D F(\bar{x}, \bar{y})(h)$ be chosen. In view of the continuity of $D F(\bar{x}, \bar{y})(\cdot)$, it is an easy observation that there exists a sequence $z_{n} \in D F(\bar{x}, \bar{y})\left(h_{n}\right)$ that converges to $\bar{z}^{1}$ :

$0=d\left(\bar{z}^{1}, D F(\bar{x}, \bar{y})(h)\right)=\lim _{n \rightarrow \infty} d\left(\bar{z}^{1}, D F(\bar{x}, \bar{y})\left(h_{n}\right)\right)$, which imply that there exist $z_{n} \in D F(\bar{x}, \bar{y})\left(h_{n}\right)$ such that $0=\lim _{n \rightarrow \infty}\left\|z_{n}-z\right\|$.

We assume that the statement is true for all semi-derivative of order $i \leq k$. Given a direction $\left(h, \bar{z}^{1}, \ldots, \bar{z}^{i}\right)$, it follows from the assumption that there are sequences $h_{n} \rightarrow$ $\bar{h}, z_{n}^{1} \rightarrow \bar{z}^{1}, \ldots, z_{n}^{k} \rightarrow \bar{z}^{k}$ such that $z_{n}^{i} \in D^{i} F(\bar{x}, \bar{y})\left(h_{n}, z_{n}^{1}, \ldots z_{n}^{i-1}\right)$ for all $i \leq k$ such that We consider similarly as above $d\left(y, D^{k} F(\bar{x}, \bar{y})\left(h_{n}, z_{n}^{1} \ldots, z_{n}^{k}\right)\right)$ for arbitrary but fixed $y \in Y$. We denote $v_{n}^{k+1}=\left(h_{n}, z_{n}^{1} \ldots, z_{n}^{k}\right)$ and $v^{k+1}=\left(\bar{h}, \bar{z}^{1} \ldots, \bar{z}^{k}\right)$. Given an $\varepsilon>0$, then there exists a positive number $t_{n}$ (that can be taken smaller than $t_{n-1}$ ) for any $n$ such that

$$
\left|d\left(y, D^{k+1} F(\bar{x}, \bar{y})\left(v_{n}^{k+1}\right)\right)-d\left(y, \Delta^{k+1} F(\bar{x}, \bar{y})\left(v_{n}^{k+1}\right)\right)\right| \leq \varepsilon
$$

by the definition of a semi-derivative and $\tau$-convergence. For the same reason the following inequality holds for $n$ large enough:

$$
\left|d\left(y, D^{k+1} F(\bar{x}, \bar{y})\left(v^{k+1}\right)\right)-d\left(y, \Delta^{k+1} F(\bar{x}, \bar{y})\left(v_{n}^{k+1}\right)\right)\right| \leq \varepsilon
$$


Consequently, we obtain the estimation

$$
\left|d\left(y, D^{k+1} F(\bar{x}, \bar{y})\left(v^{k+1}\right)\right)-d\left(y, D^{k+1} F(\bar{x}, \bar{y})\left(v_{n}^{k+1}\right)\right)\right| \leq 2 \varepsilon
$$

We conclude the desired assertion since $y$ was arbitrary.

We shall show that $D F(x, y)$ has a closed graph. The assertion follows the same way for the higher order semi-derivative. Let $\left(h_{n}, z_{n}\right) \in$ graph $D F(x, y)$ and $\left(h_{n}, z_{n}\right) \rightarrow$ $(h, z)$. Suppose $z \notin D F(x, y)(h)$. Since the semi-derivatives are closed sets, we have $d(z, D F(x, y)(h))=\alpha>0$. On the other hand $d\left(z, D F(x, y)\left(h_{n}\right)\right) \rightarrow 0$ while $n \rightarrow \infty$, because $z_{n} \in D F(x, y)\left(h_{n}\right)$ and $z_{n} \rightarrow z$. This is a contradiction to the continuity of $D F(x, y)(\cdot)$.

Proposition 2.7 Let $F: X \rightrightarrows Y$ be semi-differentiable of order $k$ at $(\bar{x}, \bar{y})$. Then the semi-derivative(s) is a lower semi-continuous multifunction of the direction, i.e., whenever $V \cap D F(\bar{x}, \bar{y})(\bar{h}) \neq \emptyset$ for some open set $V \subseteq Y$, there is a neighborhood $U \subseteq X$ of $\bar{h}$, such that $V \cap D F(\bar{x}, \bar{y})(h) \neq \emptyset$ for all $h \in \bar{U}$.

Proof: We show the statement for the first-order semi-derivative; the property for the higher-order derivative follows in the same way. Assume the opposite. This means that there is an open set $V \subseteq Y$ such that $V \cap D F(\bar{x}, \bar{y})(\bar{h}) \neq \emptyset$ and for any neighborhood $U_{\varepsilon} \subseteq X$ of $\bar{h}$, there is a point $h_{\varepsilon} \in U_{\varepsilon}$ such that $V \cap D F(\bar{x}, \bar{y})\left(h_{\varepsilon}\right)=\emptyset$. Let $z \in$ $V \cap D F(\bar{x}, \bar{y})(\bar{h})$. There exists a $\delta>0$ such that $\tilde{z} \in V$ for all $\tilde{z}:\|\tilde{z}-z\| \leq \delta$. Taking $\varepsilon=1 / n$, we obtain a sequence $h_{n} \rightarrow \bar{h}$. At the same time, it holds

$$
d\left(z, D F(\bar{x}, \bar{y})\left(h_{n}\right)\right) \geq \delta \text { and } d(z, D F(\bar{x}, \bar{y})(\bar{h}))=0 .
$$

This is a contradiction to the $\tau$-continuity proved in the previous proposition.

Various differentiability concepts for finite dimensions are compared in [5, 32]. Continuous tangential approximations of multifunctions in finite dimensions, are considered in [37], where differentiability properties of the metric projection are investigated. In [5], it is shown for mappings in finite-dimensional spaces that such tangential approximations, if they exist, coincide with the semi-derivatives, where at that place the semiderivative is understood with respect to the Kuratowski-Painlevé convergence of the differential quotients. Furthermore, in [5, 32], it is shown that proto-differentiability, resp. directional differentiability for pseudo-Lipschitzian multifunctions is equivalent to semi-differentiability.

It is not difficult to establish the latter relation also in our setting.

Recall that a set-valued mapping $F: X \rightrightarrows Y$ is said to be pseudo-Lipschitzian at a point $(\bar{x}, \bar{y}) \in \operatorname{graph} F$ if there exist neighbourhoods $U \subseteq X$ of $\bar{x}$ and $V \subseteq Y$ of $\bar{y}$, and a positive constant $L$ such that

$$
F\left(x_{1}\right) \cap V \subseteq F\left(x_{2}\right)+L\left\|x_{1}-x_{2}\right\| B,
$$

whenever $x_{1}, x_{2} \in U$. Here $B$ stands for the closed unit ball in $Y$.

Let us recall that $F$ is pseudo-Lipschitzian at $(\bar{x}, \bar{y}) \in \operatorname{graph} F$ if and only if the 
distance function $d(\cdot, F(\cdot))$ is locally Lipschitz at $(\bar{y}, \bar{x})$. This result is formulated in [33] for finite-dimensional spaces but the proof works in the infinite-dimensional case, too, and is omitted here.

Proposition 2.8 If $F: X \rightrightarrows Y$ is pseudo-Lipschitzian at a point $(\bar{x}, \bar{y}) \in \operatorname{graph} F$ and directionally differentiable in every direction at that point, then it is semi-differentiable at $(\bar{x}, \bar{y})$ and the semi-derivative coincide with the directional derivative.

Proof: Let sequences $\left\{h_{n}\right\}, h_{n} \in X$ and $\left\{t_{n}\right\}$ be given such that $h_{n} \rightarrow h_{0}$ and $t_{n} \downarrow 0$. We denote by $U$ and $V$ the neighbourhoods of $\bar{x}$ and $\bar{y}$, respectively, such that the inequality (2) holds with some constant $L$. Let $\varepsilon>0$ be given and $y \in Y$ be an arbitrary point. Having in mind the Lipschitz behavior of the distance function, we obtain the following chain of inequalities for $n$ large enough:

$$
\begin{aligned}
& \left|d\left(y, \frac{F\left(\bar{x}+t_{n} h_{n}\right)-\bar{y}}{t_{n}}\right)-d\left(y, F^{\prime}(\bar{x}, \bar{y})\left(h_{0}\right)\right)\right| \\
& \leq\left|d\left(y, \frac{F\left(\bar{x}+t_{n} h_{0}\right)-\bar{y}}{t_{n}}\right)-d\left(y, F^{\prime}(\bar{x}, \bar{y})\left(h_{0}\right)\right)\right|+ \\
& \left|d\left(y, \frac{F\left(\bar{x}+t_{n} h_{0}\right)-\bar{y}}{t_{n}}\right)-d\left(y, \frac{F\left(\bar{x}+t_{n} h_{n}\right)-\bar{y}}{t_{n}}\right)\right| \\
& \leq \varepsilon+t_{n}^{-1}\left[d\left(\bar{y}+t_{n} y, F\left(\bar{x}+t_{n} h_{n}\right)\right)-d\left(\bar{y}+t_{n} y, F\left(\bar{x}+t_{n} h_{0}\right)\right)\right] \\
& \leq \varepsilon+t_{n}^{-1} L t_{n}\left\|h_{n}-h_{0}\right\| \leq(1+L) \varepsilon
\end{aligned}
$$

Consequently, $d\left(y, t_{n}^{-1}\left[F\left(\bar{x}+t_{n} h_{n}\right)-\bar{y}\right]\right)$ converges to $d\left(y, F^{\prime}\left(\bar{x}, \bar{y} ; h_{0}\right)\right)$, which proves the assertion.

Proposition 2.9 Suppose that $F: X \rightrightarrows Y$ is pseudo-Lipschitzian around some point $(x, y) \in \operatorname{graph} F$ with a constant $L$ and semi-differentiable at that point. Then the semi-derivative at $(x, y)$ is Lipschitzian multifunction of the direction with the same Lipschitz constant.

Proof: Let $z \in D F\left(x, y ; h^{1}\right)$. Given sequences $t_{n} \downarrow 0, h_{n}^{1} \rightarrow h^{1}, h_{n}^{2} \rightarrow h^{2}$, there exist points $y_{n}^{1} \in F\left(x+t_{n} h_{n}^{1}\right)$ such that $\left(y_{n}^{1}-y\right) / t_{n} \rightarrow z$. Consider $y_{n}^{2} \in F\left(x+t_{n} h_{n}^{2}\right)$ such that $d\left(y_{n}^{1}, y_{n}^{2}\right) \leq L t_{n}\left\|h_{n}^{1}-h_{n}^{2}\right\|+\varepsilon t_{n}^{2}$ which exists by the pseudo-Lipschitz behavior of $F$ around $(x, y)$. The following estimation holds for $n$ large enough:

$$
\begin{aligned}
d\left(z, \frac{F\left(x+t_{n} h_{n}^{2}\right)-y}{t_{n}}\right) & \leq d\left(z, \frac{y_{n}^{2}-y}{t_{n}}\right) \leq d\left(z, \frac{y_{n}^{1}-y}{t_{n}}\right)+\frac{1}{t_{n}} d\left(y_{n}^{1}, y_{n}^{2}\right) \\
& \leq d\left(z, \frac{y_{n}^{1}-y}{t_{n}}\right)+L\left\|h_{n}^{1}-h_{n}^{2}\right\|+\varepsilon t_{n}
\end{aligned}
$$

Letting $n \rightarrow \infty$ we get $d\left(z, D F\left(x, y ; h^{2}\right)\right) \leq L\left\|h^{1}-h^{2}\right\|$.

Analogously, $d\left(z, D F\left(x, y ; h^{1}\right)\right) \leq L\left\|h^{1}-h^{2}\right\|$ for each $z \in D F\left(x, y ; h^{2}\right)$.

Estimating the distance of $0 \in D F(x, y ; 0)$ to $D F(x, y ; h)$ we obtain the following:

Corollary 2.10 Suppose that $F: X \rightrightarrows Y$ is pseudo-Lipschitzian around some point $(x, y) \in$ graph $F$ with a constant $L$ and semi-differentiable at that point.

Then $D F(x, y ; h) \cap B(0, L\|h\|) \neq \emptyset$. 


\section{$3 \quad$ Higher order directionally differentiable selections}

The main idea of the constructions presented in this section is to exploit the semiderivative of the multifunction, properties of the metric projections, and Michael's selection theorem.

Let us denote the metric projection of $y \in Y$ onto a closed convex set by $\operatorname{Pr}(y, A)$. Recall that

$$
\operatorname{Pr}(y, A)=\{z \in A:\|z-y\|=d(y, A)\} .
$$

First we cite some results concerning the continuity properties of the metric projection. At this place, another topology on the hyperspace $\mathcal{F}(Y)$ becomes useful. Let $m$ denotes the Mosco-topology on $\mathcal{F}(Y)$.

A sequence $\left\{A_{n}\right\}$ of weakly closed subsets of $Y$ converges to a set $A \subseteq Y$ in $(\mathcal{F}(Y), m)$ if both of the following conditions are met:

(i) for each $y \in A$ there are points $y_{n} \in A_{n}$ such that $\left\{y_{n}\right\}$ converges in norm to $y$;

(ii) whenever points $y_{k} \in A_{n_{k}}, k=1,2, \ldots$, are given, then the weak convergence of $\left\{y_{n}\right\}$ to $y \in Y$ implies $y \in A$.

This convergence is introduced by Mosco ([30]) as a modification of the sequential characterization of Kuratowski-Painlevé convergence (valid in a first countable space) in order to take into consideration the weak topology.

Recall that $Y$ has the Kadec-Klee property if weak convergence and convergence of the norms imply norm convergence. The following relation between the Mosco- and the Wijsman-topology is known:

Lemma 3.1 ([7]) Let $Y$ be a normed linear space. Then $\tau=m$ on $\mathcal{F}_{c}(Y)$ if and only if $Y$ is reflexive and the dual space $Y^{*}$ has the weak* Kadec-Klee property, i.e., weak convergence in $Y^{*}$ and convergence of the norms imply norm convergence in $Y^{*}$.

A reflexive Banach space with a strongly convex norm and the Kadec-Klee property is called an E-space. We will use the following result:

Theorem 3.2 ([21]) Let $Y$ and its dual $Y^{*}$ be E-spaces and $A, A_{n} \in \mathcal{F}_{c}(Y), n=$ $1,2, \ldots$ Then $\lim _{n \rightarrow \infty} \operatorname{Pr}\left(y_{n}, A_{n}\right)=\operatorname{Pr}(y, A)$, whenever $m-\lim _{n \rightarrow \infty} A_{n}=A$ and $\lim _{n \rightarrow \infty} y_{n}=y$.

The metric projection onto a nonempty closed convex set is unique provided that the norm is strongly convex. It is shown in [21] that the Mosco-topology is the weakest one that guarantees strong continuity of the metric projection onto moving convex sets.

Examples of E-spaces are Euclidean spaces, Hilbert spaces, uniformly convex Banach spaces (see e.g.[21]). Each reflexive space is potentially an E-space, since it can be renormed with an equivalent locally uniformly convex norm by a theorem of Troyanski (cf.[19], Theorem 1, p.164). 
Corollary 3.3 Let $Y$ and its dual $Y^{*}$ be E-spaces. Furthermore, let the multifunction $F: X \rightrightarrows Y$ be $\tau$-continuous at $\bar{x}$ and have nonempty closed convex images. Suppose that the single-valued mapping $y: X \rightarrow Y$ is continuous at $\bar{x}$ with respect to the norms in $X$ and $Y$. Then the metric projection $f(x)=\operatorname{Pr}(y(x), F(x))$ is continuous at $\bar{x}$ with respect to the norms.

Proof: The statement follows immediately from the cited Theorem 3.2 and the fact that Mosco and Wijsman topologies agree on $\mathcal{F}_{c}(Y)$.

Theorem 3.4 Let both $Y$ and $Y^{*}$ be E-spaces. Suppose $F: X \rightrightarrows Y$ to be a $\tau$-continuous multifunction with non-empty closed convex images. Furthermore, suppose that the points $x_{i} \in X, i \in I$, where $I$ is some index-set, are such that

(i) there is an $\varepsilon>0$ with $B\left(x_{i}, \varepsilon\right) \cap B\left(x_{j}, \varepsilon\right)=\emptyset$ for all $i \neq j$,

(ii) for all $i \in I$, there is a $y_{i} \in F\left(x_{i}\right): F$ is semi-differentiable of order $k$ at $\left(x_{i}, y_{i}\right)$.

Then there is a continuous selection $f$ of $F$, which satisfies the conditions: for all $i \in I, f\left(x_{i}\right)=y_{i}$ and $f$ is Hadamard-directionally differentiable of order $k$ at $x_{i}$ with $f^{\prime}\left(x_{i} ; h\right) \in D F\left(x_{i}, y_{i}\right)(h)$, for all $h \in X$.

Proof: Let $\left(x_{i}, y_{i}\right) \in$ graph $F$ be one of the chosen points. We define a neighborhood $U_{i}$ by setting $U_{i}=x_{i}+\varepsilon B$. Here $B$ is the open unit ball in $X$ and $\varepsilon$ is the positive number of assumption (a). By virtue of Proposition 2.7, $D F\left(x_{i}, y_{i} ; \cdot\right)$ is a lower semi-continuous set-valued mapping. It is easy to see that it has closed and convex images. By Michael's selection theorem, there exists a continuous selection $\bar{z}_{i}^{1}(h) \in D F\left(x_{i}, y_{i}\right)(h)$. Let us consider its restriction $\bar{z}_{i}^{1}(\cdot)$ to the unit sphere. Furthermore, $D^{2} F(\bar{x}, \bar{y})\left(h, \bar{z}_{i}^{1}(h)\right)$ is a lower semi-continuous mapping of $h$ on the closed unit ball. It has closed and convex images and, consequently, we may apply Michael's selection theorem, again. We choose a continuous selection $\bar{z}_{i}^{2}(\cdot)$ of $D^{2} F(\bar{x}, \bar{y})\left(\cdot, \bar{z}_{i}^{1}(\cdot)\right)$ on the unit sphere. Proceeding the same way, we end up with a continuous selection $\bar{z}_{i}^{k}$ of $D^{k} F(\bar{x}, \bar{y})\left(h, \bar{z}_{i}^{1}(h), \ldots, \bar{z}_{i}^{k-1}\right)$ for $h$ on the unit sphere. We observed that $z_{i}(h) \in D^{k} F(\bar{x}, \bar{y})\left(h, z_{i}^{1}, \ldots, z_{i}^{k-1}\right)$ if and only if $\lambda^{k} z_{i} \in D^{k} F(\bar{x}, \bar{y})\left(h, \lambda z_{i}^{1}, \ldots, \lambda^{i-1} z_{i}^{k-1}\right)$. Therefore, we define $z_{i}^{j}: X \rightarrow Y$ for $j=2, \ldots, k$ by setting

$$
z_{i}^{j}(h)= \begin{cases}\|h\|^{j} z_{i}^{-}(h /\|h\|) & \text { if } h \neq 0 \\ 0 & \text { if } h=0\end{cases}
$$

It is easy to see that $z_{i}^{j}(h) \in D^{j} F(\bar{x}, \bar{y})\left(h, z_{i}^{1}, \ldots, z_{i}^{j-1}\right)$ for $j=2, \ldots, k$. We choose points that are metric projections of $y_{i}+z_{i}^{1}\left(x-x_{i}\right)+\ldots+z_{i}^{k}\left(x-x_{i}\right)$ onto $F(x)$ and define

$$
f_{i}(x)=\operatorname{Pr}\left(y_{i}+z_{i}^{1}\left(x-x_{i}\right)+\ldots+z_{i}^{k}\left(x-x_{i}\right), F(x)\right) .
$$


Having in mind Corollary 3.3, it is clear that $f_{i}(\cdot)$ is a continuous function.

We consider further the set $U^{c}=X \backslash\left(\cup_{i \in I} U_{i}\right)$. We define

$$
\hat{F}(x)= \begin{cases}F(x) & \text { if } x \in U^{c}, \\ \left\{f_{i}(x)\right\} & \text { if } x \in U_{i}, i \in I .\end{cases}
$$

The definition is nonambigous since $U_{i} \cap U_{j}=\emptyset$ for $i \neq j, i, j \in I$ by the assumption (i). The mapping $\hat{F}(\cdot)$ is lower semi-continuous since so is $F$ and it has closed convex images. Applying the Michael's selection theorem, we obtain a continuous selection $f$ of $F$ such that $f(x)=f_{i}(x)$ if $x \in U_{i}$.

It remains to prove that $f$ is Hadamard-directionally differentiable of order $k$ at $x_{i}, i \in I$. By definition, this is equivalent to the statement that $f_{i}(x)$ is Hadamarddirectionally differentiable of order $k$ at $x_{i}$. Moreover, we shall show that

$$
f^{(j)}\left(x_{i}, h\right)=z_{i}^{j}(h)
$$

Given sequences $t_{n} \downarrow 0, h_{n} \rightarrow h$, let us assume that the statement is true for all derivatives of order $1, \ldots, j-1$. We shall prove it for $j$. The following chain of relations holds true:

$$
\begin{aligned}
& \left\|\frac{f_{i}\left(x_{i}+t_{n} h_{n}\right)-f_{i}\left(x_{i}\right)-t_{n} f_{i}^{(1)}\left(h_{n}\right)-\ldots-t_{n}^{j-1} f_{i}^{(j-1)}\left(h_{n}\right)}{t_{n}^{j}}-z_{i}^{j}(h)\right\| \\
= & t_{n}^{-j} \| \operatorname{Pr}\left(y_{i}+z_{i}^{1}\left(t_{n} h_{n}\right)+\ldots+z_{i}^{k}\left(t_{n} h_{n}\right), F\left(x_{i}+t_{n} h_{n}\right)\right) \\
& -y_{i}-t_{n} z_{i}^{1}\left(h_{n}\right)-\ldots-t_{n}^{j-1} z_{i}^{j-1}\left(h_{n}\right)-t_{n}^{j} z_{i}^{j}(h) \| \\
= & t_{n}^{-j} \| \operatorname{Pr}\left(y_{i}+z_{i}^{1}\left(t_{n} h_{n}\right)+\ldots+z_{i}^{k}\left(t_{n} h_{n}\right), F\left(x_{i}+t_{n} h_{n}\right)\right) \\
& -y_{i}-t_{n} z_{i}^{1}\left(h_{n}\right)-\ldots-t_{n}^{k} z_{i}^{k}\left(h_{n}\right)+t_{n}^{j} z_{i}^{j}\left(h_{n}\right)+\ldots+t_{n}^{k} z_{i}^{k}\left(h_{n}\right)-t_{n}^{j} z_{i}^{j}(h) \| \\
\leq & t_{n}^{-j} d\left(y_{i}+t_{n} z_{i}^{1}\left(h_{n}\right)+\ldots+t_{n}^{k} z_{i}^{k}\left(h_{n}\right), F\left(\bar{x}+t_{n} h_{n}\right)\right) \\
& \left.+\| z_{i}^{j}\left(h_{n}\right)+\ldots+t_{n}^{k-j} z_{i}^{k}\left(h_{n}\right)-z_{i}^{j}(h)\right) \|
\end{aligned}
$$

The latter inequality follows from the triangle inequality and the fact that

$\|\operatorname{Pr}(a, A)-a\|=d(a, A)$. The first term of the expression converges to 0 by the definition of semi-derivative of order $j$, and the second term by the construction of $z_{i}^{j}(\cdot)$.

This proves that $f$ has the desired properties.

Let us note that the constructed selection is differentiable only at certain points. An assumption about the semi-differentiability of the multifunction at each point of its graph would not lead to the directional differentiability of the selection constructed in this manner. An immediate consequence of this theorem and Proposition 2.8 is the following statement:

Corollary 3.5 Let $Y$ and $Y^{*}$ be E-spaces. Suppose $F: X \rightrightarrows Y$ to be a pseudoLipschitzian multifunction with non-empty closed convex images. Let the points $x_{i} \in$ $X, i \in I$, where $I$ is some index-set, are chosen such that 
(i) there is an $\varepsilon>0$ with $B\left(x_{i}, \varepsilon\right) \cap B\left(x_{j}, \varepsilon\right) \forall i \neq j$,

(ii) for all $i \in I$, let $y_{i} \in F\left(x_{i}\right): F$ is directionally differentiable at $\left(x_{i}, y_{i}\right)$.

Then there is a continuous selection $f$ of $F$ such that for all $i \in I, f\left(x_{i}\right)=y_{i}$ and $f$ is Hadamard-directionally differentiable at $x_{i}$ with $f^{\prime}\left(x_{i} ; h\right) \in F^{\prime}\left(x_{i}, y_{i}\right)(h)$ for all $h \in X$.

\section{Remark 3.6 Renorming of the image space}

As already mentioned, every reflexive space can be renormed with an equivalent locally uniformly convex norm (see Theorem 1, p.164, and Theorem 2, p.37 in [19]). The new norm, although compatible with the original one, generates a new Wijsman topology on $\mathcal{F}(Y)$. It has been proved in [10] that, given a linear normed space $Y$ and a family $\pi$ of equivalent norms, the family of distance functionals $\left\{d_{p}(y, \cdot): y \in Y, p \in \pi\right\}$ determines a weak topology on $\mathcal{F}_{c}(Y)$ that coincides with the slice topology. We do not provide information about the latter topology because it will not be essentially for our paper and refer the interested reader to [7]. Assuming $Y$ to be a Banach space, the slice topology on $\mathcal{F}_{c}(Y)$ agrees with a Wijsman topology that is determined by a norm such that the dual norm has the weak* Kadec-Klee property. If, in addition, $Y$ is reflexive, then both topologies coincide with the Mosco topology. Therefore, we may modify the assumptions of the latter two theorems in the following way:

Corollary 3.7 Let $Y$ be a reflexive Banach space. Suppose $F: X \rightarrow \mathcal{F}_{c}(Y)$ to be $m$ continuous (equivalently: continuous with respect to the slice topology). We assume that for all $x \in X$ there is a $y \in F(x)$ such that $F$ is semi-differentiable of order $k$ at $(x, y)$. Given such a point $(\bar{x}, \bar{y})$, there exists a continuous selection $f$ of $F$ with $f(\bar{x})=\bar{y}$, which is Hadamard-directionally differentiable of order $k$ at $\bar{x}$ with derivatives

$$
f^{(j)}(\bar{x} ; h) \in D^{j} F(\bar{x}, \bar{y})\left(h, f^{(1)}(\bar{x} ; h), \ldots, f^{(j-1)}(\bar{x} ; h)\right), \forall h \in X, \forall j=1, \ldots, k .
$$

In [20], Dommisch establishes the following result on this topic. The existence of a smooth partition of unity in $\mathbb{R}^{n}$ is exploited there to prove the existence of a smooth selection in the following situations: $F$ acts from a closed subset of $\mathbb{R}^{m}$ in $\mathbb{R}^{n}$ and has convex images and open preimages, i.e., $F^{-1}(y)=\{x \in X: y \in F(x)\}$ is open in $X$ for all $y \in \mathbb{R}^{n}$. The proof of this assertion can be carried over also to the case $X$ is a weakly countably determined Banach space and $Y$ is a linear normed space.

A Banach space $X$ is called weakly countably determined if there exists a countable collection $\left\{K_{n}\right\}$ of weak* compact subsets of $X^{* *}$ such that for every $x \in X$ and $u \in X^{* *} \backslash X$ there exists an index $n$ such that $x \in K_{n}$ and $u \notin K_{n}$. We recall that, given a linear normed space $X$ and an open covering $X=\cup_{\alpha \in \Lambda} V_{\alpha}$ of it, a continuous partition of unity subordinated to $\left\{V_{\alpha}\right\}_{\Lambda}$ is a union $\left\{g_{\alpha}\right\}_{\Lambda}$ of continuous functions $g_{\alpha}$ such that:

- for all $x \in X$, it holds $0 \leq g_{\alpha}(x) \leq 1$ and $\sum_{\alpha \in \Lambda} g_{\alpha}(x)=1$,

- $\cup_{\alpha \in \Lambda}\left(\operatorname{supp} g_{\alpha}\right)=X$, 
- for any $\alpha \in \Lambda$ there exists a finite number of $\beta \in \Lambda$ such that supp $g_{\alpha} \cap \operatorname{supp} g_{\beta} \neq \emptyset$,

- for any $\alpha \in \Lambda$ one has supp $g_{\alpha} \subseteq V_{\alpha}$.

Theorem 3.8 Let $X$ be a weakly countably determined Banach space and $Y$ be a linear normed space. Assume that $F$ has nonempty closed convex images and open preimages, i.e., $F^{-1}(y)=\{x \in X: y \in F(x)\}$ is open in $X$ for all $y \in F(X)$.

Then $F$ admits Gâteaux differentiable selection.

Proof: The set $\bigcup_{y \in F(X)} F^{-1}(y)$ forms an open covering of $X$. By virtue of Corollary 3.3, Chap.VIII in [18] the countably determined Banach space $X$ admits a Gâteaux differentiable partition of unity subordinated to this covering, i.e., the functions $g$ are Gâteaux differentiable. Each function $g_{\alpha}$ corresponds to some set $F^{-1}(y)$ and we note this by writing $g_{y}$ instead of $g_{\alpha}$. We define

$$
f(x)=\sum_{y \in F(x)} g_{y}(x) y .
$$

Given a point $x, \sum_{y \in F(x)} g_{y}(x) y$ is a finite sum by the local finiteness of the partition of unity. It is a finite convex combination of the points $y \in F(x)$ such that $x \in \operatorname{supp} g_{y}$ and, thus, $f(x) \in F(x)$. We show that $f$ is Gâteaux differentiable. Let $\bar{x}$ be a given point, and $\left\{x_{n}\right\}$ be a sequence converging in norm to $\bar{x}$. By the local finiteness of the partition, there exists a neighborhood of $\bar{x}$ that has a nonempty intersection with finitely many supp $g_{y}$. Therefore, in the definition of $f\left(x_{n}\right)$ the same terms $g_{y}\left(x_{n}\right) y$ vanish for all $x_{n}$ with $n$ large enough. Consequently, $f(x)$ is Gâteaux differentiable at $\bar{x}$ with derivative $f^{\prime}(x)=\sum_{y \in F(x)} g_{y}^{\prime}(x) y$.

The assertion remains true in the situation that $\operatorname{dom} F$ is a proper closed subset of $X$. In this case, we simply have to include $X \backslash \operatorname{dom} F$ to the open covering of $X$.

The selection constructed in the latter theorem has stronger differentiability properties globally. However, the assumptions of the theorem are not satisfied when $F$ is an upper semi-continuous mapping for instance. It is known (cf.[7]) that the preimages $F^{-1}(y)$ are closed provided $F$ is upper semi-continuous.

Recall that a bump function on $X$ is a function with bounded nonempty support on $X$.

Theorem 3.9 Let $X$ be a weakly countably determined Banach space, which admits $C^{k}$-smooth bump function. Assume that $F$ has nonempty closed convex images and open preimages as in the previous theorem. Then $F$ admits a $C^{k}$-smooth selection.

Proof: The construction of the previous theorem can be used again. According to Theorem 3.2, Chap.VIII in [18] under the assumptions of the proposition the space $X$ admits a $C^{k}$-smooth partition of unity. The statement follows by the same reasons as in the previous proof. 


\section{Univalued Representations}

Definition 4.1 Given a multifunction $F$, we shall call a countable family of selections $\left\{f_{n}\right\}$ a univalued representation of it, if graph $F=c l \cup_{n=1}^{\infty}\left(\operatorname{graph} f_{n}\right)$.

Another notion of univalued representation is considered in [2] as a "almost" Castaing representation.

Theorem 4.2 Let $F: X \rightrightarrows Y$ be a $\tau$-continuous multifunction with nonempty closed convex images, which is semi-differentiable of order $m$ at all points $(x, y) \in \operatorname{graph} F$. Assume that $X$ and $Y$ are separable normed spaces and $Y$ and $Y^{*}$ are E-spaces. Then $F$ admits a univalued representation $\left\{f_{n}\right\}$ with the following property:

(D) For any point $(x, y) \in \operatorname{graph} F$ and for any $\varepsilon>0$ there is a selection $f_{n}$ such that $\left\|y-f_{n}(x)\right\| \leq \varepsilon$ and $f_{n}$ is directionally differentiable of order $m$ at some $\tilde{x}:\|x-\tilde{x}\| \leq \varepsilon$. Moreover, the collections $f_{n}^{(i)}(\tilde{x} ; \cdot)$ are univalued representations of the semiderivatives $D^{i} F\left(\tilde{x}, f_{n}(\tilde{x})\right)(\cdot)$, for all $i=1, \ldots, m$.

Proof: In a first step we shall construct selections passing through a set of points dense in the graph of $F$ and Hadamard-directionally differentiable of order $m$ at a chosen point. In a second step, we shall add countably many selections by modifying the family of the first set such that the graphs of the directional derivative of those selections are dense in the graph of the corresponding semi-derivative of $F$ at the considerate points. Let $\left\{x_{n}\right\}$ be a countable dense set of points in $X$ and $\left\{y_{n}^{i}\right\}$ be a dense set of points in $F\left(x_{n}\right)$. We use Theorem 3.4 to construct selections $f_{n i}$ such that $f_{n i}\left(x_{n}\right)=y_{n}^{i}$. Now, for each point $\left(x_{n}, y_{n}^{i}\right)$ we add another set of countably many selections $f_{j l}$ with the following properties: $f_{j l}\left(x_{n}\right)=y_{n}^{i}$ and $f_{j l}^{(1)}\left(x_{n} ; h_{l}\right)$ are dense in $D F\left(x_{n}, y_{n}^{i}\right)\left(h_{l}\right)$, where $h_{l}, l=1,2, \ldots$ are dense in $X$. In order to do this, let us consider a countable set of points $\bar{z}_{l}^{j_{1}}$ that is dense in $D F\left(x_{n}, y_{n}^{i}\right)\left(h_{l}\right)$. Such a set exists since $Y$ is separable. We can construct continuous selections $z_{l j_{1}}(\cdot) \in D F\left(x_{n}, y_{n}^{i}\right)(\cdot)$ such that $z_{l j_{1}}\left(h_{l}\right)=\bar{z}_{r}^{j_{1}}$ in the following way. We define $D F_{l j_{1}}(h)=D F\left(x_{n}, y_{n}^{i}\right)(h)$ for all $h \neq h_{l}$ and $D F_{l j_{1}}\left(h_{l}\right)=\bar{z}_{l}^{j_{1}}$. The mapping $D F_{l j_{1}}(\cdot)$ is lower semi-continuous since $D F\left(x_{n}, y_{n}^{i}\right)(\cdot)$ is also. It has convex closed images and, hence, there is a continuous selections $\hat{z}_{l j_{1}}(\cdot)$ of it by virtue of Michael's selection theorem. We use $\hat{z}_{l j_{1}}(\cdot)$ to define a mapping $z_{l j_{1}}^{1}(\cdot)$ as follows:

$$
z_{l j_{1}}^{1}(a)= \begin{cases}\frac{\|a\|}{\left\|h_{l}\right\|} \hat{z}_{l j_{1}}\left(a \frac{\left\|h_{l}\right\|}{\|a\|}\right) & \text { if } a \neq 0 \\ 0 & \text { if } a=0\end{cases}
$$

The selections $z_{l j_{1}}^{1}(\cdot)$ of $D F\left(x_{n}, y_{n}^{i}\right)(\cdot)$ have the desired properties: it is continuous, positive homogenius, and $z_{l j_{1}}^{1}\left(h_{l}\right)=\bar{z}_{l}^{j_{1}}$. In a similar way we determine points which are dense in the images of the second-order derivative in the directions $\left(h_{l}, \bar{z}_{l j_{1}}\right)$. We construct a countably many selections $z_{l j_{2}}^{2}(\cdot)$ of the second-order derivative running trough those points. We proceed this way until we construct countably many selections $z_{l j_{m}}^{m}(\cdot)$ of the semi-derivative of order $m$ with the necessary properties. Further, 
we use $z_{l j_{1}}^{1}(\cdot), \ldots, z_{l j_{m}}^{m}(\cdot)$ for constructing $f_{l j_{1} j_{2} \ldots j_{m}}$ as in the Theorem 3.4.

A multifunction satisfying the assumptions of the theorem admits a univalued representation such that for every point of the graph there is a point close to it and a selection that is Hadamard-directionally differentiable at the latter point. Under an additional assumption, we can modify the univalued representation constructed in the above theorem in such a way that it is also pointwise dense in the images of the multifunction. Moreover, the directional derivatives of the selections will form a pointwise dense representation of the semi-derivatives $D^{i} F\left(x_{n}, y_{n}^{i}\right)(\cdot)$

Definition 4.3 A set-valued mapping $F: X \rightrightarrows Y$ is said to be sub-Lipschitzian at a point $(\bar{x}, \bar{y}) \in \operatorname{graph} F$ if for any closed balls $B(\bar{x}, \alpha) \subseteq X$ and $B(\bar{y}, \beta) \subseteq Y$ there is a positive constant $L$ such that

$$
F\left(x_{1}\right) \cap B(\bar{y}, \beta) \subseteq F\left(x_{2}\right)+L\left\|x_{1}-x_{2}\right\| B(0,1),
$$

whenever $x_{1}, x_{2} \in B(\bar{x}, \alpha)$.

A notion of sub-Lipschitzian set-valued mappings has been introduced in [33]. The difference is that in [33] compact sets are used instead of the closed balls of the above definition. It is clear that the two notions coincide if $X$ and $Y$ are finite-dimensional spaces. Moreover, in these case a multifunction with nonempty closed images is subLipschitzian if and only if it is pseudo-Lipschitzian. For $X$ and $Y$ being infinitedimensional the sub-Lipschitz behavior is, obviously, a stronger property than the pseudo-Lipschitz behavior.

let us recall the following result:

Lemma 4.4 ([29], Lemma 5.2.) If $X$ is perfectly normal, $Y$ is separable Banach space, and if $F: X \rightrightarrows Y$ is lower semi-continuous, then there exists a countable collection of selections $\left\{f_{n}\right\}$ such that, for every $x \in X,\left\{f_{n}(x)\right\}$ is dense in $F(x)$.

A normal space is called perfectly normal, if every closed subset is a $G_{\delta}$, i.e., each metric space is perfectly normal.

Theorem 4.5 Under the conditions the previous theorem, suppose, in addition, that $F$ is sub-Lipschitzian. Then $F$ admits a univalued representation with the property (D) and such that:

$$
F(x)=c l \cup_{n=1}^{\infty} f_{n}(x) \text { and } D F\left(x_{n}, y_{n}^{i}\right)(h)=c l \cup_{l=1}^{\infty}\left\{f_{l}^{\prime}\left(x_{n} ; h\right): f_{l}\left(x_{n}\right)=y_{n}^{i}\right\}
$$

for $h \neq 0$ and for set of points $\left\{x_{n}\right\}$ dense in $x,\left\{y_{n}^{i}\right\}$ dense in $F\left(x_{n}\right)$.

Proof: We shall slightly modify the construction of the previous theorem in the following way. Let $L_{n i}$ be the smallest Lipschitz constant associated with the point $\left(x_{n}, y_{n}^{i}\right)$ and its neighborhood $U_{n} \times V_{n}^{i} \subseteq X \times Y$. Moreover, let the neighborhood be 
chosen such that $U_{n} \subseteq B\left(x_{n}, 1\right)$ and $V_{n}^{i} \subseteq B\left(y_{n}^{i}, 1\right)$. We make use of the Corollary 2.10 and a consider multifunction $D F_{n i}(h):=D F\left(x_{n}, y_{n}^{i}\right)(h) \cap B\left(0, L_{n i}+1\right)$ for $\|h\| \leq 1$. This multifunction has nonempty closed convex images and is lower semi-continuous, because $D F\left(x_{n}, y_{n}^{i}\right)(\cdot)$ is as well. Therefore, by virtue of Lemma 4.4 there is a countable family of selections

$\operatorname{bar} z_{n i}^{1 j}$ whose images are pointwise dense in the images of $D F_{n i}(\cdot)$. We consider the restrictions of those selections to the unit sphere. It holds $\left\|\bar{z}_{n i}^{1 j}(h)\right\| \leq L_{n i}+1$. We proceed further as in the previous proof to construct a countable family $\left\{f_{l}\right\}$ using only the first order semi-derivative, i.e., we apply the construction for the case $m=1$. This set of selections is added to the the univalued representation of $F$ constructed in the previous theorem. It is obvious, that property $(\mathrm{D})$ is satisfied. We have to show the pointwise density in the images of $F$ and in the images of $D F\left(x_{n}, y_{n}^{i}\right)$.

Claim 1: $F(x)=c l \cup_{l} f_{l}(x)$ for all $x \in X$.

Let $(x, y) \in \operatorname{graph} F$ and $\delta>0$. By the density of $\left\{x_{n}\right\}$ in $X$, and of $\left\{y_{n}^{i}\right\}$ in $F\left(x_{n}\right)$, for any $\varrho>0$ there exist points $x_{k}$ such that $\left\|x-x_{k}\right\|<\varrho$ and $y_{k}^{i} \in F\left(x_{k}\right)$ such that $\left\|y_{k}^{i}-\operatorname{Pr}\left(y, F\left(x_{k}\right)\right)\right\|<\varrho$. Since $Y$ is an E-space the notation $\operatorname{Pr}\left(y, F\left(x_{k}\right)\right)$ makes sense. By construction, there is a selection $f_{k i}$ with $f_{k i}\left(x_{k}\right)=y_{k}^{i}$. Recall that $f_{k i}$ is constructed in such a way that

$$
f_{k i}(x)=\operatorname{Pr}\left(y_{k}^{i}+z_{k i}\left(x-x_{k}\right), F(x)\right)
$$

for $k$ large enough. Using this, the sub-Lipschitzian behavior of $F$, and the properties of the metric projection, we obtain:

$$
\begin{aligned}
\left\|y-f_{k i}(x)\right\| & \leq\left\|y-\operatorname{Pr}\left(y, F\left(x_{k}\right)\right)\right\|+\left\|\operatorname{Pr}\left(y, F\left(x_{k}\right)\right)-y_{k}^{i}\right\|+\left\|y_{k}^{i}-f_{k}^{i}(x)\right\| \\
& \leq \varrho L+\varrho \\
& +\left\|\operatorname{Pr}\left(y_{k}^{i}+z_{k i}\left(x-x_{k}\right), F(x)\right)-\operatorname{Pr}\left(y_{k}^{i}, F\left(x_{k}\right)\right)\right\| .
\end{aligned}
$$

Since $\varrho$ can be chosen arbitrarily small, the first and second term of the right hand-side in the last inequality will become smaller than $\delta$. We have to show that this holds also for the last term. By virtue of the Lemma 3.3 the metric projections converge uniformly if the projected points converge in norm and the sets converge in the sense of Mosco. These conditions will be satisfied if we can show that $\left\|z_{k i}\left(x-x_{k}\right)\right\|$ converges to zero while $k \rightarrow \infty$. By construction $\left\|\bar{z}_{k i}\left(\frac{x-x_{k}}{\left\|x-x_{k}\right\|}\right)\right\| \leq L_{k i}+1$. Remember that the constants $L_{n i}$ were taken to be the smallest Lipschitz constants associated with the neighbourhoods $U_{n} \times V_{n}^{i}$ of the points $\left(x_{n}, y_{n}^{i}\right)$. We consider the closed set $B(x, 1+$ $\left.\varrho_{0}\right) \times B\left(y, 1+\varrho_{0}\right)$ for some $\varrho_{0}>0$. It contains the whole neighbourhoods $U_{k} \times V_{k}^{i}$ for $\varrho<\varrho_{0}$. By the definition of sub-Lipschitzian multifunctions, there is a Lipschitz constant $\bar{L}$ associated with that set and it is evident that $L_{n i} \leq \bar{L}$. Therefore, it holds:

$$
\left\|x-x_{k}\right\| \cdot\left\|\bar{z}_{k i}\left(\frac{x-x_{k}}{\left\|x-x_{k}\right\|}\right)\right\| \leq \varrho(\bar{L}+1)
$$

Letting $\varrho \rightarrow 0$ we obtain the desired estimate.

Claim 2: $D F\left(x_{n}, y_{n}^{i}\right)(h)=c l \cup\left\{f_{l}^{\prime}\left(x_{n} ; h\right): f_{l}\left(x_{n}\right)=y_{n}^{i}\right\}$ for all $h \in X, h \neq 0$. 
By construction $f_{l}^{\prime}\left(x_{n} ; h\right) \in D F\left(x_{n}, y_{n}^{i}\right)(h)$ for all selections $f_{l}$ of the collection for which it holds $f_{l}\left(x_{n}\right)=y_{n}^{i}$. Moreover, $f_{l}^{\prime}\left(x_{n} ; h\right)$ are dense in $D F\left(x_{n}, y_{n}^{i}\right)(h)$ for $\|h\|=1$ by construction according to Lemma 4.4 . Let $h$ be arbitrary point of $X$, different from $0, z \in D F\left(x_{n}, y_{n}^{i}\right)(h)$, and $\varepsilon>0$. We denote $\lambda:=1 /\|h\|$. It holds $\lambda z \in D F\left(x_{n}, y_{n}^{i}\right)(\lambda h)$ because the semi-derivative is a process. There exists a selection $f_{l}$ such that $\left\|f_{l}^{\prime}\left(x_{n} ; \lambda h\right)-\lambda z\right\|<\varepsilon \lambda$ since $\|\lambda h\|=1$. By the positive homogeneity of $f_{l}^{\prime}$, it holds $\left\|f_{l}^{\prime}\left(x_{n} ; h\right)-z\right\|<\varepsilon$, which proves the assertion.

Unfortunately, the directional derivatives of the selections are not pointwise (i.e. for each direction) dense in the corresponding whole semi-derivative, that is the semiderivative calculated at any point of the graph of the multifunction. What conditions on the mapping imply a continuity of the semi-derivatives with respect to the point they are calculated at, is an open question. Moreover, if such conditions are identified, there is still the question, whether those conditions imply also the pointwise density of the collections of the single-valued directional derivatives considered above.

Let us emphasize, that we can construct the selections also in such a way, that their higher order directional derivative are pointwise dense in the images of the higher order semi-derivatives at those points $\left(x_{n}, y_{n}^{i}\right)$, excluding the directions starting with the zero element of $X$.

\section{Measurability and Delta-Theorems}

We equip the linear normed space $X$ with a $\sigma$-algebra $\mathcal{A}$. The Borel $\sigma$-algebra will be denoted by $\mathcal{B}(X)$. The following definition of measurability will be used (see also $[7,4])$ :

Definition 5.1 A mapping $f:(X, \mathcal{A}) \rightarrow(Y, \mathcal{B}(X))$ is measurable if for any open set $G \subseteq Y$ the preimage $f^{-1}(G)=\{x \in X: f(x) \in G\}$ belongs to $\mathcal{A}$.

A set-valued mapping $F:(X, \mathcal{A}) \rightrightarrows(Y, \mathcal{B}(X))$ is measurable if for any open set $G \subseteq Y$ $F^{-1}(G)=\{x \in X: F(x) \cap G \neq \emptyset\} \in \mathcal{A}$.

Recall that $f:(X, \mathcal{A}) \rightarrow Y$ is called a measurable selection of $F$ if $f$ is measurable and $f(x) \in F(x)$ almost surely.

It is a fundamental result (cf. [26]) that a closed-valued measurable multifunction in a Polish image space admits a measurable selection. Furthermore, for a multifunction $F$ with non-empty closed values in a Polish target space we can choose a countable family of measurable selections $\left\{f_{n}\right\}$ that pointwise fills up the values of the multifunction:

$$
\text { for each } x \in X, F(x)=c l\left(\cup_{n=1}^{\infty} f_{n}(x)\right) \text {. }
$$

Such a countable family is called a Castaing representation for the multifunction. The existence of such a representation characterizes measurability. 
Our aim is to construct Castaing representations of a multifunction $F: X \rightrightarrows Y$ with additional differentiability properties. This investigation is motivated on the one hand by the natural idea to construct representation that is a kind of expansion of the multifunction. On the other hand such a representation is of interest while working with the delta method. Delta theorems are concerned with the asymptotic distribution of functions of random elements. The following theorem is known.

Theorem 5.2 ([40]) Suppose that $X$ and $Y$ are Banach spaces and let $f:(X, \mathcal{B}(X)) \rightarrow$ $(Y, \mathcal{B}(Y))$ be measurable and Hadamard-directionally differentiable at some point $\bar{x} \in$ $X$. Suppose that $t_{n}\left(x_{n}-\bar{x}\right)$ are some random elements of $X$ converging in distribution to some element $z$, written

$$
t_{n}^{-1}\left(x_{n}-\bar{x}\right) \stackrel{D}{\rightarrow} h,
$$

while $t_{n} \downarrow 0$ and $h$ is a random element in some separable subspace of $X$. Then

$$
t_{n}\left(f\left(x_{n}\right)-f(\bar{x})\right) \stackrel{D}{\rightarrow} f^{\prime}(\bar{x} ; h) .
$$

Here $\stackrel{D}{\rightarrow}$ denotes convergence in distribution.

Recall that convergence in distribution of a sequence of random elements $x_{n}$,

$x_{n}:(\Omega, \mathcal{A}, P) \rightarrow X$, means the weak* convergence of the measures $\mu_{n}=P \circ x_{n}^{-1}$ that these elements induce on the space $X$. A sequence of probability measures $\mu_{n}$ on a separable metric space $X$ weakly* converges to $\mu$ if one of the following equivalent conditions is satisfied (cf.[12]):

(i) $\liminf _{n} P\left\{x_{n} \in G\right\} \geq P\{x \in G\}$ for all open sets $G$,

(ii) $\limsup _{n} P\left\{x_{n} \in C\right\} \leq P\{x \in C\}$ for all closed sets $C$,

(iii) $\lim _{n \rightarrow \infty} \int g(x) \mu_{n}(d x)=\int g(x) \mu(d x)$ for all bounded continuous functions $g: X \rightarrow \mathbb{R}$.

A closed-valued multifunction $F:(\Omega, \mathcal{A}) \rightrightarrows(Y, \mathcal{B}(Y))$ can be identified with a single valued mapping $\tilde{F}:(\Omega, \mathcal{A}) \rightarrow(\mathcal{F}(Y), \mathcal{B}(\mathcal{F}(Y)))$. As already mentioned, if $(Y, d)$ is Polish, then $(\mathcal{F}(Y), \tau)$ is Polish, too. We consider the Borel sigma algebra on $\mathcal{F}(Y)$ generated by the Wijsman topology and ask whether the measurability of $\tilde{F}$ is related to the measurability of $F$. The answer to this question is due to Hess (see [24]): Assuming $Y$ to be separable, $F$ is measurable if and only if $\tilde{F}$ is as well. Then, in a natural way, convergence in distribution of $F_{n}:(\Omega, \mathcal{A}) \rightrightarrows Y$ to some $F:(\Omega, \mathcal{A}) \rightrightarrows Y$ can be understood as the weak* convergence of the measures induced on $\mathcal{F}(Y)$ by $\tilde{F}_{n}$. Convergence in distribution of set-valued mappings with images in $\mathbb{R}^{n}$ is considered in [34] and some investigations for the infinite-dimensional case are given in [35].

The first set-valued version of a delta-theorem was formulated by King [25] for random sets in $\mathbb{R}^{n}$. Corresponding results for random sets in infinite dimensions seem not to be known. There is also no characterization for the asymptotic behavior of the ensemble 
of measurable selections. Let us emphasize that the distribution of a random set does not determine the ensemble of distributions of its measurable selections (see [1] also the references therein). In [1] the set of distributions of measurable selections of random sets has been studied from a different point of view. The primary object there is a given probability distribution on some compact subset of a complete separable metric space. The problem which distributions on the space are induced by selections of random sets with the the given probability distribution is investigated.

Now we are ready to prove a generalized delta-theorem for random sets in infinitedimensional spaces. Moreover, using the results of the previous section, we show deltatheorem for all measurable selection of a Castaing representation of the sets.

Theorem 5.3 Let $X$ be a separable Banach space and $Y$ be a Polish space. Suppose that $F: X \rightrightarrows Y$ is a measurable multifunction that is semi-differentiable at the point $(\bar{x}, \bar{y})$. Let us assume that the random elements $x_{n}:(\Omega, \mathcal{A}) \rightarrow X$ satisfy a generalized central limit formula with limit $\bar{x}$, i.e.,

$$
t_{n}^{-1}\left(x_{n}-\bar{x}\right) \stackrel{D}{\rightarrow} h
$$

while $t_{n} \downarrow 0$ and $h$ is a random element in some separable subspace of $X$.

Given a point $\bar{y} \in F(\bar{x})$, then $F$ satisfies the central limit formula in the sense that

$$
t_{n}^{-1}\left(F\left(x_{n}\right)-\bar{y}\right) \stackrel{D}{\rightarrow} D F(\bar{x}, \bar{y})(h)
$$

as random sets in $\mathcal{F}(Y)$.

If the additional assumptions of Theorem 4.2 are satisfied, then $F$ admits a Castaing representation $\left\{f_{k}\right\}$ such that all $f_{k}$ satisfy the generalized central limit formula

$$
t_{n}^{-1}\left[f_{k}\left(x_{n}\right)-f_{k}(\bar{x})\right] \stackrel{D}{\rightarrow} f_{k}^{\prime}(\bar{x} ; h) \in D F\left(\bar{x}, f_{k}(\bar{x})\right)(h)
$$

as random variables in $Y$. Moreover, $f_{k}^{\prime}(\bar{x} ; h)$ are dense in $D F(\bar{x}, \bar{y})(h)$ and there is an index $k$ such that $f_{k}(\bar{x})=\bar{y}$.

Proof: We have to show the convergence in distribution of the random sets. Let $\mu_{n}$ and $\mu$ be the corresponding probability measures induced on $X$ by $h_{n}:=t_{n}^{-1}\left(x_{n}-\bar{x}\right)$ and by $h$, respectively. $F$ is measurable and, therefore, the corresponding mapping $\tilde{F}: X \rightarrow$ $\mathcal{F}(Y)$ is measurable. Let us consider the random elements $g_{n}\left(h_{n}\right):=t_{n}^{-1}\left(\tilde{F}\left(x_{n}\right)-\bar{y}\right)$ and $g(h)=D F(\bar{x}, \bar{y})(h)$ in $\mathcal{F}(Y)$. The measures induced on the Polish space $\mathcal{F}(Y)$ by these elements are $\mu_{n} \circ g_{n}^{-1}$ and $\mu \circ g^{-1}$. According to Theorem 5.5 in [12] the central limit formula $\left(\mu_{n} \circ g_{n}^{-1} \rightarrow \mu \circ g^{-1}\right)$ will be proved if $\lim _{n \rightarrow \infty} g_{n}\left(h_{n}\right)=g(h)$ holds for $\mu$-almost all points $h$ and $h_{n}$ such that $h_{n}$ approaches $h$. This is true by the definition of semi-differentiability.

The existence of a Castaing representation with the desired differentiability p property follows from Theorem 4.2. We can simplify the construction in the following way. In the second step, we only need to consider points $\bar{z}_{j}$ that is dense in $D F(\bar{x}, \bar{y})(h)$, and 
add continuous selections $z_{j}(\cdot) \in D F(\bar{x}, \bar{y})(\cdot)$ such that $z_{j}(h)=\bar{z}_{j}$.

A relevant result is contained in the paper [27]. A multifunction acting between two infinite-dimensional spaces is considered there. The values $F(x)$ are supposed to be compact and $F(\bar{x})=\bar{y}$ to be a singleton. The statement says that for a measurable selections of $F$ there is a subsequence satisfying the formula holds.

Assuming higher-order differentiability for the multifunction, we are able to obtain higher-order information on the asymptotic behavior.

Theorem 5.4 Let $X$ be a separable Banach space and $Y$ be a Polish space. Suppose that $F: X \rightrightarrows Y$ is a measurable multifunction. Let us assume that the random elements $x_{n}:(\Omega, \mathcal{A}) \rightarrow X$ satisfy a generalized central limit formula with limit $\bar{x}$, i.e.,

$$
t_{n}^{-1}\left(x_{n}-\bar{x}\right) \stackrel{D}{\rightarrow} h
$$

while $t_{n} \downarrow 0$ and $h$ is a random element in some separable subspace of $X$. Furthermore, let a point $\bar{y} \in F(\bar{x})$ be given. Denote $h_{n}=t_{n}^{-1}\left(x_{n}-\bar{x}\right)$.

(i) Assume that $F$ is semi-differentiable at $(\bar{x}, \bar{y})$ of order $k$ and the mappings $z^{1}(\cdot)$, $z^{2}(\cdot), \ldots, z^{k-1}(\cdot)$ are continuous selections of the semi-derivatives Then $F$ satisfies the following higher-order central limit formula:

$t_{n}^{-k}\left[F\left(x_{n}\right)-\bar{y}-t_{n} z^{1}\left(h_{n}\right)-\ldots-t_{n}^{k-1} z^{k-1}\left(h_{n}\right)\right] \stackrel{D}{\rightarrow} D^{k-1} F(\bar{x}, \bar{y})\left(h, z^{1}(h), \ldots, z^{k-1}(h)\right)$

as random sets in $\mathcal{F}(Y)$,

(ii) Suppose the assumptions of Theorem 4.5. If $F$ is semi-differentiable of order $k$ at all points $(\bar{x}, y)$ with $y \in F(\bar{x})$, then $F$ admits a Castaing representation $\left\{f_{j}\right\}$ such that all $f_{j}$ satisfy the higher-order central limit formula

$$
t_{n}^{-k}\left[f_{j}\left(x_{n}\right)-f_{j}(\bar{x})-t_{n} f_{j}^{(1)}\left(\bar{x} ; h_{n}\right)-\ldots-t_{n}^{k-1} f_{j}^{(k-1)}\left(\bar{x} ; h_{n}\right)\right] \stackrel{D}{\rightarrow} f_{j}^{(k)}(\bar{x} ; h)
$$

as random variables on $Y$.

Moreover, $f_{j}^{(k)}(\bar{x} ; h)$ are dense in $D^{k} F(\bar{x}, \bar{y})\left(h, z^{1}(h), \ldots, z^{k-1}(h)\right)$, and there is an index $l$ such that $f_{l}(\bar{x})=\bar{y}$.

Proof: The line of arguments of the previous proof can be applied to this case, too, with the only difference that higher-order differentiable selections are used to construct the Castaing representation.

Let $\mu_{n}$ and $\mu$ be the corresponding probability measures induced on $X$ by $h_{n}=t_{n}^{-1}\left(x_{n}-\right.$ $\bar{x})$ and by $h$, respectively. We consider the random elements $g_{n}\left(h_{n}\right)=t_{n}^{-k}\left(\tilde{F}\left(x_{n}\right)-\bar{y}-\right.$ $\left.t_{n} z^{1}\left(h_{n}\right)-\ldots-t_{n}^{k-1} z^{k-1}\left(h_{n}\right)\right)$ and $g(h)=D^{k} F(\bar{x}, \bar{y})\left(h, z^{1}(h), \ldots, z^{k-1}(h)\right)$ in $\mathcal{F}(Y)$. The measures induced on the Polish space $\mathcal{F}(Y)$ by these elements are $\mu_{n} \circ g_{n}^{-1}$ and $\mu \circ g^{-1}$. One has $\mu_{n} \circ g_{n}^{-1} \rightarrow \mu \circ g^{-1}$ if $\lim _{n \rightarrow \infty} g_{n}\left(h_{n}\right)=g(h)$ holds for $\mu$-almost all points 
$h$ and $h_{n}$ such that $h_{n} \rightarrow h$. This is true by the given definition of semi-differentiability of order $k$.

Let $\left\{x_{k}\right\}$ be a countable dense set of points in $X$ and $\left\{y_{n}^{j}\right\}$ be a dense set of points in $F\left(x_{k}\right)$. Furthermore, let the points $\bar{y}^{j}$ form a countable dense set in $F(\bar{x})$ and $\bar{y}$ belong to this set. We shall construct selections $f_{k j}$ such that $f_{k j}\left(x_{k}\right)=y_{k}^{j}$ and $f_{k j}(\bar{x})=\bar{y}^{j}$. We make sure that $x_{k} \neq \bar{x}$ for all $k=1,2, \ldots$. Since the points $x_{k}$ and $\bar{x}$ can always be separated by neighbourhoods, we may use Theorem 3.4 to construct $f_{k j}$. We only slightly modify the construction as it was done in the proof of Theorem 4.5.

The asymptotic properties of the selections follow by similar arguments as above. In order to see this, define $g_{n j}\left(h_{n}\right)=t_{n}^{-k}\left[f_{j}\left(x_{n}\right)-f_{j}(\bar{x})-t_{n} f_{j}^{(1)}\left(\bar{x} ; h_{n}\right)-\ldots-t_{n}^{k-1} f_{j}^{(k-1)}\left(\bar{x} ; h_{n}\right)\right]$ and $g_{j}(h)=f_{j}^{(k)}(\bar{x} ; h)$. We have $\lim _{n \rightarrow \infty} g_{n j}\left(h_{n}\right)=g_{j}(h)$ for all $h$ by the Hadamarddirectional differentiability of $f_{k}$ of order $k$ at $\bar{x}$. The statement follows by virtue of the Theorem 5.5 in [12].

Summarizing we can say that we do not have a result stating a central limit formula for all selections of the multifunction, but we are able to construct a Castaing representation with this property under relatively general assumptions. Having in mind the literature mentioned above, it is clear that statements of this kind should be possible for all measurable selections under very restrictive assumptions only.

\section{References}

[1] Z. Artstein, Distributions of random sets and random selections, Israel J. Math. 46 (1984) 313-324

[2] Z. Artstein, On dense univalued representations of mulivalued maps, Rendiconti der Circolo Mathematico di Palermo, Serie II, Tomo XXXIII, (1983) 340-350

[3] J.-P. Aubin and A. Cellina, Differential Inclusions, Springer, Grundlehren der math. Wiss., 1984, Vol.264.

[4] J.-P. Aubin and H. Frankowska, Set-Valued Analysis, Birkhäuser, Boston 1990.

[5] A. Auslender and R. Cominetti, A comparative study of multifunction differentiability with applications in mathematical programming, Mathematics of Operations Research, 16 (1991), 240-258.

[6] H.T.Banks and M.Q. Jacobs, A differential calculus for multifunctions, Journal of Mathematical Analysis and Applications, 29 (1970) 246-272.

[7] G. Beer, Topologies on Closed and Closed Convex Sets, Kluwer, Mathematics and its Applications, Vol.268, 1993

[8] G. Beer, Wijsman convergence: a survey, Set-Valued Analysis 2 (1994) 77-94 . 
[9] G. Beer, A Polish topology for the closed subsets of a Polish space, Proc. Amer. Math. Soc. 113(1991), 1123-1133.

[10] G. Beer, Wijsman convergence of convex sets under renorming, Nonlinear Analysis, Theory, Methods\& Applications, 22 (1994) 2, 207-216.

[11] A. Ben-Tal and J. Zowe, A unified theory for first- and second-order conditions for extremum problems in topological vector spaces, Mathematical Programming Study 19 (1982) $39-76$

[12] P. Billingsley, Convergence of probability measures, Wiley, New York, 1968.

[13] Ch. Castaing, Sur les multi-application measurables, Inf. Rech. Op. 1 (1967) 91-126

[14] Ch. Castaing and M. Valadier, Convex analysis and measurable multifunctions, Springer, Lecture Notes in Math., 1977, Vol.580.

[15] R. Cominetti, Metric regularity, tangent sets and second-order optimality conditions, Applied Mathematics and Optimization 21 (1990), 265-287.

[16] F.S.De Blasi, On the differentiability of multifunctions, Pacific J.Mathematic, 66 (1976) $67-81$.

[17] D. Dentcheva, Differentiable selections and Castaing representations of multifunctions, Journal of Mathematical Analysis and Applications, 223 (1998) 371-396.

[18] R. Deville, G. Godefroy and V. Zizler, Smoothness and renorming in Banach spaces, Longman Scientific \& Technical, 1993.

[19] J. Diestel, Geometry of Banach spaces, Lecture Notes in Mathematics Vol.485, SpringerVerlag, Berlin, 1975.

[20] G. Dommisch, On the existence of Lipschitz-continuous and differentiable selections for multifunctions, in J.Guddat et al.(eds.): Parametric Optimization and related topics, Math. Res. Vol. 35, Akademie-Verlag Berlin, 1987, 60 - 73.

[21] A. Dontchev and T. Zollezzi, Well-posed optimization problems, Lecture Notes in Mathematics Vol.1543, Springer-Verlag, Berlin, 1993.

[22] S. Gautier, Affine and eclipsing multifunctions, Numer. Funct. Anal. and Optimiz. 11(7\& 8) (1990), 679-699.

[23] S. Gautier and R. Morchadi, A selection of convex-compact-valued multifunctions with remarkable properties: the Steiner selection, Numer. Funct. Anal. and Optimiz. 13(5\& 6) (1992) 513-522

[24] C. Hess, Quelques resultats sur la measurabilité des multifunctions à valeurs dans un espace métrique séparable, Séminaire d'Analyse Convexe de l'Université de Montpellier, Exposé 1, 1986.

[25] A.J. King, Generalized delta theorems for multivalued mappings and measurable selections, Mathematics of Operations Research 14 (1989) 4, 720-736 
[26] K. Kuratowski and C. Ryll-Nardzewski, A general theorem on selectors, Bull. Acad. Pol. Sc. 13, 1965, 397-403.

[27] P. Lachout, On multifunction transforms of probability measures, Annals of Operations Research, 56 (1995) 241-250.

[28] C. Lemaréchal and J. Zowe, The eclipsing concept to approximate a multi-valued mapping. Optimization 63 (1956) 233-237.

[29] E. Michael, Continuous selections I, Annals of Mathematics 63 (1956) 2, 361-382.

[30] U. Mosco, Convergence of convex sets and solutions of variational inequalities, Adv. in Math. 3 (1969), 510-585.

[31] J.-P. Penot, Differentiability of relations and differential stability of perturbed optimization problems, SIAM J. Control and Optimization 22 (1984) 4, 529-551

[32] R. T. Rockafellar, Proto-differentiability of set-valued mappings and its application in optimization, in: H. Attouch et al. (eds.), Analyse Non Lineaire, Gauthier-Villars, Paris, 1989, 449-482.

[33] R. T. Rockafellar, Lipschitzian properties of multifunctions, J. Nonlinear Analysis: Theory, Mathods, Applications 9 (1985) 867-885.

[34] G. Salinetti and R.J.-B. Wets, On the convergence in distribution of measurable multifunctions (random sets), normal integrands, stochastic processes and stochastic infima, Mathematics of Operations Research 11 (1986) 3, 385-419.

[35] G.Salinetti and R.J.-B.Wets, Weak convergence of probability measures revisited, IIASA Working paper WP-87-30, Laxenburg, 1987.

[36] A. Seeger, Second-order directional derivatives in parametric optimization, Mathematics of Operations Research 13 (1988) 124-139.

[37] A. Shapiro, Existence and differentiability of metric projections in Hilbert spaces, SIAM J. Optimization 4 (1994) 1, 130-141.

[38] A. Shapiro, On differentiability of metric projections in $\mathbb{R}^{n}$ : Boundary Case, Proc.Amer. Math. Sic. 99, 123-128.

[39] A. Shapiro, Directional differentiability of metric projections onto moving sets at boundary points, Journal of Mathematical Analysis and Applications 13 (1988) 392-403.

[40] A. Shapiro, Asymptotic analysis of stochastic programs, Annals of Operations Research 30 (1991), 169-186.

[41] R.A. Vitale, The Steiner point in infinite dimensions, Israel Journal of Mathematics 52 (1985) 245-250. 